\title{
Treatment of ventricular tachycardia: consider ablation sooner
}

\section{Heather L Bloom}

\author{
Address: Division of Cardiovascular Medicine, Emory University School of Medicine and Atlanta VA Medical Center, \\ 1670 Clairmont Rd, Decatur, GA 30033, USA \\ Email: hbloom@emory.edu
}

FI000 Medicine Reports 2009, I:7I (doi:I0.34I0/MI-7I)

The electronic version of this article is the complete one and can be found at: http://FI000.com/Reports/Medicine/content/I/7I

\begin{abstract}
Ventricular tachycardia (VT) is a leading cause of morbidity and mortality for many patients, with a significant emotional and economic burden caused by implantable cardioverter-defibrillator (ICD) shocks and the requirement of medication with significant side effects. Additionally, $10 \%$ of VT occurs in patients with no structural heart disease. Until quite recently, ablation for VT has been reserved as the procedure of last hope for those who have ongoing recurrences despite maximal medical therapy and who are traumatized by multiple ICD shocks [I]. However, recent advances in imaging technology and three-dimensional intracardiac mapping systems have significantly improved the safety and efficacy of VT ablation procedures. Thus, ablation for VT should no longer be reserved as a lastresort bailout procedure and should move into the realm of routine electrophysiology treatment.
\end{abstract}

\section{Introduction and context}

Clinical management of ventricular arrhythmias (VAs) is problematic and often complicated by the need for rapid diagnosis and treatment. Monomorphic ventricular tachycardia (VT) is most commonly due to re-entry and is commonly seen in patients with underlying structural heart disease. There is typically a zone of slow conduction due to scarring or inhomogeneous myocardium. Causes include prior infarct, primary cardiomyopathy, surgical scarring, hypertrophy, and muscle degeneration. Underlying pathologies include sarcoid, Chagas disease, dilated cardiomyopathy, valvular heart disease, or arrhythmogenic right ventricular dysplasia [1-5]. Standard treatment of VAs due to these pathologies has been beta-blockers, anti-arrhythmic drugs, and implantation of implantable cardioverter-defibrillators (ICDs). Beta-blockers are the only medication with proven efficacy in reducing sudden cardiac death, although anti-arrhythmics may be effective as an adjunctive therapy to reduce VA burden and prevent ICD therapies [6]. Historically, mapping VTs in the electrophysiology (EP) lab for ablation in cardiomyopathic patients has been problematic due to hemodynamic instability. Advances in imaging and three-dimensional (3D) mapping systems, leading to improved substrate definition, have allowed significant improvements in these procedures for these patients.

Additionally, $10 \%$ of monomorphic VT occurs in patients with structurally normal hearts [7]. Mechanistically, these VTs are more often due to triggered activity or abnormal automaticity. Although patients with idiopathic VTs usually have a good prognosis, sudden death is still known to occur and pharmacological treatments have poor efficacy and high rates of side effects. Most, if not all, of these atypical VTs are amenable to ablation, which has been considered first-line therapy for these patients [6]. Recent advances in technology and techniques, and new data supporting the safety and efficacy of palliation in numerous disease states, support performing ablation more routinely in patients with structural heart disease.

\section{Ablation of ventricular tachycardia: historical perspective Historically, electrophysiologists had only the tools of pace-mapping, entrainment, and activation mapping to attempt ablation of VT. Pace-mapping refers to assessing the activation sequence of the VT by 12-lead}


electrocardiogram in the EP lab during pacing. The site where the paced QRS morphology matches the clinical VT pattern is likely to be the site of origin for a focal VT, or the exit site for scar-related VT. Activation mapping refers to the method of moving an electrode catheter point by point through multiple ventricular sites during VT and recording the electrogram at each site. The site of earliest activation (that is, the earliest onset of electrical depolarization) identifies the origin of the tachycardia, or exit site. Entrainment mapping is used for re-entry circuits and consists of pacing during VT at a rate faster than the tachycardia, from a site thought to be within the circuit. Examining the change in QRS morphology during pacing and the post-pacing timing of resumption of the VT helps determine whether the paced site is within the re-entry circuit. Both activation and entrainment mapping require a hemodynamically stable arrhythmia to allow these manipulations. All three techniques are tedious and time-consuming. Another confounding problem is that multiple morphologies of VT often can be induced, leading to uncertainty as to which is the clinical arrhythmia.

\section{Recent advances}

\section{Advances in ablation technique}

Within the last decade, attention has turned to substrate mapping. Substrate mapping uses our understanding of the pathophysiology of re-entrant arrhythmias to characterize areas in the myocardium likely to sustain re-entry based on anatomic features or electrophysiological characteristics (for example, a scar can be identified by bipolar electrogram amplitude). Electroanatomic mapping systems create a 3D computer-generated image of the ventricular geometry. These are either contact or non-contact systems that consist of electromagnetic fields that record intracardiac electrical activation points from catheter electrodes. They display a map of the anatomy with the intracardiac catheters superimposed, and this map can reduce fluoroscopic radiation exposure. They also can produce voltage maps of the myocardium which define areas of ventricular scar based on absolute voltage. Substrate-based mapping allows ablation lines to be drawn from areas of scar to electrically inactive areas to cut off potential re-entrant loops, potentially avoiding the need to do maneuvers during sustained VT.

\section{Advances in imaging}

\section{Magnetic resonance imaging}

Magnetic resonance imaging (MRI) identifies non-viable myocardium, focal fibrosis, and peri-infarct zone volume via late gadolinium enhancement. In multiple disease processes, this non-viable myocardium has proven to be predictive of VT prevalence or mortality or both. MRI- defined border zone function has been demonstrated to correlate with inducibility of VT [8]. MRI clearly delineates the location of scar tissue, which often corresponds to critical areas within VT circuits. This detailed anatomic knowledge allows pre-procedure planning of ablation strategy, suggesting how lines can be drawn to link nonviable myocardium with electrically neutral structures such as valves. Software also allows incorporation of the MRI image into the electroanatomic map in the lab for real-time image correlation with substrate. Unfortunately, MRI cannot be performed in patients with pacemakers or ICDs, limiting its utility immensely $[9,10]$.

Positron emission tomography - computed tomography

Ablation of scar-related VT is facilitated by accurate definition of the scar borders. Positron emission tomography (PET) imaging is extremely accurate in defining 3D scar anatomy. Two recent studies demonstrated that PET - computed tomography (CT) scans performed pre-procedure can also be integrated into the 3D mapping system in the EP lab and registered to the voltage electroanatomic map. Thus, simultaneous display of the PET-CT scar and border zone information can be incorporated into the in-lab voltage map. This improves accuracy of the in-lab map and allows simultaneous visualization of the anatomic image plus substrate to facilitate identification of critical areas to target for VT ablation [11,12].

\section{Recent data on ablation efficacy and safety in multiple disease processes}

Ablation of ventricular tachycardia in patients with ischemic and non-ischemic cardiomyopathy

The SCD-HeFT (Sudden Cardiac Death in Heart Failure Trial) investigators reviewed their patients who received primary prevention ICDs. Patients who had VT had a significant increase in the risk of death compared with those who did not experience VT (hazard ratio 5.7, $P<0.001)$ [13]. A study published in The New England Journal of Medicine in December 2007 randomly assigned patients with coronary artery disease and sustained VTs undergoing ICD implantation to substrate-based catheter ablation versus non-invasive interventions (medication) [14]. Patients on the ablation arm had less ICD therapy for VA (hazard ratio $0.35, P=0.007$ ) and a nonsignificant trend toward lower mortality ( $9 \%$ versus $17 \%, P=0.29)[14]$. There were no deaths from the ablation procedure or evidence of adverse events. These data support the contention that catheter ablation is useful as a palliative therapy for malignant VAs in patients with primary prevention ICDs, and suggest that further investigation may reveal improvements in mortality after VT ablation. The University of Michigan recently reviewed their experience with mapping and 
ablation for 35 non-ischemic VT patients ablated in 2007. Patients who failed endocardial ablation underwent a second procedure with transcutaneous epicardial mapping and ablation. The authors report a 60-70\% success rate in controlling VTs refractory to medical management and a major complication rate of less than 5\% [15]. Carbucicchio and colleagues [16] have demonstrated improved mortality outcomes when VT ablation is offered to extremely sick patients in VT storm. The authors prospectively evaluated 95 patients with cardiomyopathy and electrical storm. At the 22-month follow-up, 92\% were free of electrical storm and $66 \%$ were free of VT recurrence [16]. Stevenson and colleagues [17] evaluated 231 patients with ischemic cardiomyopathy and recurrent VT. Ablation was carried out in all patients with an electroanatomic mapping system to assist substrate mapping during sinus rhythm. At the 6-month follow-up, 53\% of patients were free of VT, and the 6-month frequency of VT episodes was reduced from a median of 11.5 to 0 in the 142 patients who had ICDs. A total of seven deaths were recorded within 7 days of the procedure, and the 1-year mortality rate was $18 \%$ [17].

\section{Ventricular tachycardia ablation in cardiac sarcoid}

In a multicenter registry, 42 patients with a diagnosis of cardiac sarcoidosis were identified and followed. When VT occurred, the patients first received ICD placement, immunosuppressive agents, anti-arrhythmic medications, and then radio frequency (RF) ablation. Nine patients were brought for ablation procedures and a total of 44 VTs were induced. Endocardial RF ablation was performed in eight patients, and epicardial RF ablation was performed in one patient. Elimination was successful in 31 of $44(70 \%)$ VTs, resulting in a decrease from 271 to 4 episodes of VT. All patients had either a decrease or a complete elimination of VT during a mean follow-up of 19.8 months. The authors conclude that catheter ablation of VT in sarcoid patients can significantly reduce VT burden [18].

\section{New anatomic sites}

\section{Catheter ablation in the sinus of Valsalva}

VAs can come from the endocardium or the epicardium. Epicardial arrhythmias arising from the aortic valve and the aortic sinus of Valsalva have been described by several groups. Rillig and colleagues [19] examined a group of 15 patients who underwent ablation in the aortic sinus of Valsalva for VT or premature ventricular contractions (PVCs). The authors reported success in 13 of 15, with recurrence and success in a second ablation procedure in the other 2 patients. There were no major complications, leading the authors to conclude that ablation within the sinus of Valsalva is a safe and effective approach for VT/PVCs [19].

\section{Epicardial ablation}

For both ischemic and non-ischemic cardiomyopathy, up to one-third of VT circuits are inaccessible from the endocardium. Percutaneous pericardial access allows for many of these VTs to be ablated from the epicardial service. This requires percutaneous access to the pericardium in the subxyphoid region. Although this procedure does carry risks of injury to epicardial coronary arteries, the phrenic nerve, subdiaphragmatic vessels, and the right ventricle, experienced centers are finding the procedure to be safe and well tolerated. Grimard and colleagues [20] last month published a review of 35 epicardial procedures attempted in 32 patients. The authors obtained an immediate successful ablation of the clinical VT in 22 of $29(76 \%)$ cases. During a mean followup of $384 \pm 405$ days, 9 patients (26\%) experienced a recurrence of a sustained VT. One patient died from tamponade during the procedure. The authors concluded that the safety and efficacy were similar to those of endocardial ablation procedures [20].

\section{Implications for clinical practice}

The presence of VAs clearly increases risk of mortality and is a significant cause of morbidity. VT episodes can be terminated, but not prevented, using an ICD. In patients with these devices, anti-arrhythmic drug therapy is often administered to prevent VT; however, these agents have adverse effects and relatively poor efficacy. Patients who receive ICD shocks have significant impairment of quality of life. Although the ability to permanently eliminate VT is determined by the substrate, recent evidence shows significant benefits in many patients, regardless of the underlying pathology. Improvements in technologies to assist with VT ablation, including improved imaging systems, 3D electroanatomic mapping systems that allow for substrate mapping in hemodynamically compromised patients, and integration of pre- and peri-procedure images, have vastly improved the safety and efficacy of these techniques. New ablation sites, including the epicardium, also show great promise for future improvements in efficacy. Many centers have provided data on the safety and efficacy of VT ablation for a variety of pathological conditions. Thus, ablation of VT should be moved out of the realm of a treatment of last resort and should be considered sooner. We have demonstrated that we have the technology not only to palliate the sickest patients, but to improve, and potentially even save, many lives.

\section{Abbreviations}

3D, three-dimensional; CT, computed tomography; EP, electrophysiology; ICD, implantable cardioverterdefibrillator; MRI, magnetic resonance imaging; PET, positron emission tomography; PVC, premature 
ventricular contraction; RF, radio frequency; SCD-HeFT, Sudden Cardiac Death in Heart Failure Trial; VA, ventricular arrhythmia; VT, ventricular tachycardia.

\section{Competing interests}

The author declares that she has no competing interests.

\section{References}

I. Sacher F, Tedrow UB, Field ME, Raymond JM, Koplan BA, Epstein LM, Stevenson WG: Ventricular tachycardia ablation: evolution of patients and procedures over $\mathbf{8}$ years. Circ Arrhythm Electrophysiol 2008, I:|53-6I.

2. Eckart RE, Hruczkowski TW, Tedrow UB, Koplan BA, Epstein LM, Stevenson WG: Sustained ventricular tachycardia associated with corrective valve surgery. Circulation 2007, I I6:2005-I I.

3. Hsia $\mathrm{HH}$, Marchlinski FE: Electrophysiology studies in patients with dilated cardiomyopathies. Card Electrophysiol Rev 2002, 6:472-81.

4. Koplan BA, Soejima K, Baughman K, Epstein LM, Stevenson WG: Refractory ventricular tachycardia secondary to cardiac sarcoid: electrophysiologic characteristics, mapping, and ablation. Heart Rhythm 2006, 3:924-9.

5. Marchlinski FE, Zado E, Dixit S, Gerstenfeld E, Callans DJ, Hsia $H$, Lin D, Nayak H, Russo A, Pulliam W: Electroanatomic substrate and outcome of catheter ablative therapy for ventricular tachycardia in setting of right ventricular cardiomyopathy. Circulation 2004, I I 0:2293-8.

6. European Heart Rhythm Association; Heart Rhythm Society, Zipes DP, Camm A], Borggrefe M, Buxton AE, Chaitman B, Fromer M, Gregoratos G, Klein G, Moss AJ, Myerburg RJ, Priori SG, Quinones MA, Roden DM, Silka MJ, Tracy C, Smith SC Jr., Jacobs AK, Adams CD, Antman EM, Anderson JL, Hunt SA, Halperin JL, Nishimura R, Ornato JP, Page RL, Riegel B, Priori SG, Blanc JJ et al .: American College of Cardiology; American Heart Association Task Force; European Society of Cardiology Committee for Practice Guidelines: ACC/AHA/ESC 2006 guidelines for management of patients with ventricular arrhythmias and the prevention of sudden cardiac death: a report of the American College of Cardiology/American Heart Association Task Force and the European Society of Cardiology Committee for Practice Guidelines (Writing Committee to Develop Guidelines for Management of Patients With Ventricular Arrhythmias and the Prevention of Sudden Cardiac Death). J Am Coll Cardiol 2006, 48:e247-346.

7. Klein LS, Shih HT, Hackett FK, Zipes DP, Miles WM: Radiofrequency catheter ablation of ventricular tachycardia in patients without structural heart disease. Circulation 1992, 85:1666-74.

8. Fernandes VR, Wu KC, Rosen BD, Schmidt A, Lardo AC, Osman N, Halperin HR, Tomaselli G, Berger R, Bluemke DA, Marbán E, Lima JA: Enhanced infarct border zone function and altered mechanical activation predict inducibility of monomorphic ventricular tachycardia in patients with ischemic cardiomyopathy. Radiology 2007, 245:7। 2-9.

FI000 Factor 3.0 Recommended

Evaluated by Arthur Stillman 17 Dec 2007

9. Nazarian S, Bluemke DA, Halperin HR: Applications of cardiac magnetic resonance in electrophysiology. Circ Arrhythm Electrophysiol 2009, 2:63-71.

FI000 Factor 6.0 Must Read

Evaluated by Heather Bloom 17 Apr 2009

10. Adabag AS, Maron BJ, Appelbaum E, Harrigan CJ, Buros JL, Gibson CM, Lesser JR, Hanna CA, Udelson JE, Manning WJ, Maron MS: Occurrence and frequency of arrhythmias in hypertrophic cardiomyopathy in relation to delayed enhancement on cardiovascular magnetic resonance. I Am Coll Cardiol 2008, 5 I:I369-74.

FI000 Factor 3.0 Recommended

Evaluated by Robert Rho 13 Mar 2009

II. Fahmy TS, Wazni OM, Jaber WA, Walimbe V, Di Biase L, Elayi CS, Difilippo FP, Young RB, Patel D, Riedlbauchova L, Corrado A, Burkhardt JD, Schweikert RA, Arruda M, Natale A: Integration of positron emission tomography/computed tomography with electronantomical mapping: a novel approach for ablation of scar-related ventricular tachycardia. Heart Rhythm 2008, 5: I538-45.

12. Tian J, Smith MF, Chinnadurai P, Dilsizian V, Turgeman A, Abbo A, Gajera K, Xu C, Plotnick D, Peters R, Saba M, Shorofsky S, Dickfeld T: Clinical application of PET/CT Fusion Imaging for ThreeDimensional Myocardial Scar and Left Ventricular Anatomy during Ventricular Tachycardia Ablation. J Cardiovasc Electrophysiol 2008, [Epub ahead of print].

13. Poole JE, Johnson GW, Hellkamp AS, Anderson J, Callans DJ, Raitt MH, Reddy RK, Marchlinski FE, Yee R, Guarnieri T, Talajic M, Wilber DJ, Fishbein DP, Packer DL, Mark DB, Lee KL, Bardy GH: Prognostic importance of defibrillator shocks in patients with heart failure. N Engl J Med 2008, 359:1009-17.

Changes Clinical Practice

FI000 Factor 6.6 Must Read

Evaluated by Hector Ventura 03 Oct 2008, Brian Olshansky 09 Oct 2008, Melvin Cheitlin 02 Jan 2009

14. Reddy VY, Reynolds MR, Neuzil P, Richardson AW, Taborsky M, Jongnarangsin K, Kralovec S, Sediva L, Ruskin JN, Josephson ME: Prophylactic catheter ablation for the prevention of defibrillator therapy. N Engl J Med 2007, 357:2657-65.

Changes Clinical Practice

FI000 Factor 9.0 Exceptional

Evaluated by Jesus Almendral II Feb 2008

15. Bogun F, Morady F: Ablation of ventricular tachycardia in patients with nonischemic cardiomyopathy. J Cardiovasc Electrophysiol 2008, 19:1227-30.

16. Carbucicchio C, Santamaria M, Trevisi N, Maccabelli G, Giraldi F, Fassini G, Riva S, Moltrasio M, Cireddu M, Veglia F, Della Bella P: Catheter ablation for the treatment of electrical storm in patients with implantable cardioverter-defibrillators: shortand long-term outcomes in a prospective single center study. Circulation 2008, II 7:462-9.

Changes Clinical Practice

FI000 Factor 6.4 Must Read

Evaluated by Paulus Kirchhof I 9 Feb 2008, Jesus Almendral I 9 Sep 2008

17. Stevenson WG, Wilber DJ, Natale A, Jackman WM, Marchlinski FE, Talbert T, Gonzalez MD, Worley SJ, Daoud EG, Hwang C, Schuger C, Bump TE, Jazayeri M, Tomassoni GF, Kopelman HA, Soejima K, Nakagawa H; Multicenter Thermocool VT Ablation Trial Investigators: Irrigated radiofrequency catheter ablation guided by electroanatomic mapping for recurrent ventricular tachycardia after myocardial infarction: the multicenter thermocool ventricular tachycardia ablation trial. Circulation 2008, I I 8:2773-82.

18. Jefic D, Joel B, Good E, Morady F, Rosman H, Knight B, Bogun F: Role of radiofrequency catheter ablation of ventricular tachycardia in cardiac sarcoidosis: report from a multicenter registry. Heart Rhythm 2009, 6:189-95.

19. Rillig A, Meyerfeldt U, Birkemeyer R, Treusch F, Kunze M, Brasch M, Jung W: Catheter ablation within the sinus of Valsalva-a safe and effective approach for treatment of atrial and ventricular tachycardias. Heart Rhythm 2008, 5:1265-72.

FI000 Factor 3.0 Recommended

Evaluated by Jack Kron I2 Dec 2008

20. Grimard C, Lacotte J, Hidden-Lucet F, Duthoit G, Gallais Y, Frank R: Percutaneous Epicardial Radiofrequency Ablation of Ventricular Arrhythmias After Failure of Endocardial Approach: A 9-Year Experience. J Cardiovasc Electrophysiol 2009, [Epub ahead of print]. 